\title{
Efficient routing in multicast mesh by using forwarding nodes and weighted cost function
}

\author{
Kapila Vyas $^{1 *}$, Ajay Khuteta ${ }^{1}$ and Amit Chaturvedi ${ }^{2}$ \\ ${ }^{1}$ Poornima University, \\ Jaipur- India \\ [e-mail: kapilapareek@yahoo.co.in] \\ ${ }^{2}$ Government engineering College \\ Ajmer - India \\ *Corresponding author: Kapila Vyas
}

Received December 23, 2018; revised February 17, 2019; revised April 6, 2019; revised May 7, 2019; accepted June 4, 2019; published December 31, 2019

\begin{abstract}
Multicast Mesh based Mobile Ad-hoc NETworks (MANETs) provide efficient data transmission in energy restraint areas without a fixed infrastructure. In this paper, the authors present an improved version of protocol SLIMMER developed by them earlier, and name it SLIMMER-SN. Most mesh-based protocols suffer from redundancy; however, the proposed protocol controls redundancy through the concept of forwarding nodes. The proposed protocol uses remaining energy of a node to decide its energy efficiency. For measuring stability, a new metric called Stability of Node (SN) has been introduced which depends on transmission range, node density and node velocity. For data transfer, a weighted cost function selects the most energy efficient nodes / most stable nodes or a weighted combination of both. This makes the node selection criteria more dynamic. The protocol works in two steps: (1) calculating SN and (2) using SN value in the weighted cost function for selection of nodes.

The study compared the proposed protocol, with other mesh-based protocols PUMA and SLIMMER, based on packet delivery ratio (PDR), throughput, end-to-end delay and average energy consumption under different simulation conditions. Results clearly demonstrate that SLIMMER-SN outperformed both PUMA and SLIMMER.
\end{abstract}

Keywords: Multicast mesh, Energy efficient protocol, Node stability 


\section{Introduction}

Mobile Ad Hoc Networks (MANETs) are most helpful in situations which are power deficient and cannot have a fixed infrastructure, as in case of natural and manmade disasters. MANETs consist of mobile nodes, each of which also works as a router. Multicast mesh based routing creates alternate routes between source and destination reducing the bandwidth consumption, traffic and delay, supporting group-oriented applications and increasing robustness [1, 2].

However, this redundancy is obtained at a cost of increased energy consumed. So the need to provide services to customers while preserving energy is an important challenge for routing in multicast. As traditional routing cannot suffice to continuous changing topology of MANETs, another important challenge in mobile ad-hoc networks is to provide stable routes [3]. Several routing protocols have been proposed to maintain energy efficiency and link stability in ad-hoc networks $[4,5]$.

Link stability measures amount of time a link can withstand in a network while continuing to send data, without any breakage [6].

Various approaches were proposed for link stability in MANETs. Most of these use either node's mobility $[7,8,9]$ or its residual energy to find stable links [10, 11].In some cases a combination of parameters was also used [4, 12]. Node Stability-based Routing used mobility degree of a node relative to its neighbours, to establish stable and sustainable paths $[7,8]$. Another approach used link lifetime prediction method using transmission range and location parameters (position, speed and direction) of the sending node and neighbouring node [9]. A recent approach using both energy and relative velocity of nodes, for computing acceptability of a path from a multicast sender to a multicast receiver was given in [25]. Energy Efficient Lifetime Aware Multicast (EELAM) worked by selecting optimal intermediate nodes with maximal residual energy [10].

Link-stability and Energy aware Routing protocols (LAER) approach tried to optimize a route using link stability and minimum drain rate energy [11]. A routing strategy using energy-rich nodes to provide efficient route was proposed by authors in [12]. Another solution for optimization problem of the MANETs using transmission range and number of nodes was given in [13]. A reliable and energy-aware multicast ad hoc on demand distance vector (REA-MAODV) routing protocol was proposed which could find energy-efficient multicast routes from source node to a group of destination node[26]. Efficient power aware routing (EPAR) method proposed recently, selects nodes on the basis of residual battery power, and the expected energy consumed for forwarding data on a specific link[27].

It is evident from the literature, that a major challenge in case of MANETs is to provide an efficient stable route for energy restraint, continuously mobile nodes. The current paper proposes a mesh based multicast protocol called Stable Links in Multicast Mesh for Energy Efficient Routing using stable nodes (SLIMMER-SN) to meet these requirements. The major contribution of the paper is that unlike its predecessors it does not broadcast packets throughout the network, but selects an Energy Efficient Stable connected dominating set (EESCDS) to do so. The CDS [18, 19, and 20] is selected by using an algorithm based on self pruning algorithm given by Lim and Kim [22].The proposed protocol controls flooding and avoids redundancy, congestion, undue energy expenditure and relatively gives better link stability. Another important contribution is that the algorithm uses a weighted cost function which can favour selection of (a) energy efficient nodes in conditions requiring high energy consumptions and/or (b) stable nodes in highly dynamic conditions, by allocation of suitable 
weights. Extensive simulations were carried out on ns 2.35 which showed that SLIMMER-SN outperformed both PUMA and SLIMMER in terms of PDR, throughput, delay and average energy consumed.The rest of the paper is organized as follows: Section 2 discusses the background of SLIMMER-SN and related studies; Section 3 presents the proposed protocol SLIMMER-SN; Section 4 presents the experimental results and Section 5 concludes the study and presents the future work.

\section{Related Work}

Our protocol is close to Protocol for Unified Multicasting through Announcement (PUMA) [14] and Stable Links in Multicast Mesh for Energy Efficient Routing (SLIMMER) [15]. The protocol SLIMMER-SN uses the concept of a cost factor based on energy efficiency and stability of nodes to find efficient routes, which has been further detailed in the following sub-sections.

A. Protocol for Unified Multicasting through Announcement (PUMA)

PUMA is a receiver initiated multicast mesh protocol. It uses the same multicast announcement (MA) for election of cores, for deciding the routes for sources outside a multicast mesh, to unicast data packets toward the group, and for maintaining mesh. It thus achieves a higher data delivery ratio with lower overheads.

Each node maintains a connectivity list for every multicast group, by storing the information from MAs which it receives from its neighbours. A multicast mesh is established, rooted at the core, with initially all receivers as mesh members. A non-receiver could be a mesh member if it lies on one of the shortest paths, from a receiver to the core. Data packets are forwarded to a mesh member in unicast manner, and then flooded within the mesh.An important constraint in PUMA is that the next forwarding nodes for construction of mesh, are selected on hop counts, which could result in link breaks if (i) the node moves away out of transmission range, as could be in high mobility network or (ii) if it becomes passive due to energy restraints. Further, flooding of data cause:

(1) Congestion and packet loss and

(2) Loss of energy in nodes employed in large scale indiscriminate flooding.

SLIMMER improves on PUMA by employing nodes which are energy-efficient to become mesh members. Although many different works were proposed for improving PUMA but they did not discuss much about energy efficiency or stability [16, 17].

\section{B. Stable Links in Multicast Mesh for Energy Efficient Routing (SLIMMER)}

Stable Links in Multicast Mesh for Energy Efficient Routing (SLIMMER) was proposed by the authors as an energy efficient improvement over PUMA. Unlike PUMA, which only considers hop count as decision criteria for making mesh, only an energy efficient node can be a next hop in SLIMMER. SLIMMER uses the remaining energy of a node to be the measure of the energy efficiency of that node. In SLIMMER Ek >= Ethresh, is a condition necessary for a node $k$ to be energy efficient where Ek is the remaining energy of the node, Ethresh is threshold energy selected for all the nodes in a network and is taken as a positive fraction of initial energy of the node.Nodes with least distance from core and with energy more than selected threshold energy are to be used as next hops.

So for SLIMMER the remaining energy $\mathrm{E}$ of a node $k$ for a time period of $t$, is defined as: 
Ek $=$ Ekinit - Ekcons

Where

Ekinit is the initial energy of a node $k$ at time $t$

Ekcons is the total energy consumed by a node $k$

Given as:

Ekcons $=$ Ektrans. nk trans + Ekrecv. nkrecv + Eksleep + Ekidle

Where

Eksleep is the energy consumed by a node $k$ in sleep mode.

Ekidle is the energy consumed by a node $k$ in idle mode.

nk trans $=$ no. of packets transmitted by node $k$ in time $t$.

Ektrans $=$ energy consumed by a node $k$ in transmitting one packet.

nkrec $=$ no. of packets received by $k$ in time $t$.

Ekrec $=$ energy consumed by a node $k$ in receiving one packet

\section{Energy efficiency of a node}

Energy efficiency of a node in this study, was measured in terms of the remaining energy as in SLIMMER. The current study decides energy efficiency of a node on the basis of its (i) distance from the core and (ii) residual energy.

Thus energy-efficient nodes are those with (a) shortest distance to the core and (b) and fulfilling the necessary condition of $\mathrm{E}>=$ Ethresh. This contributes in avoiding futile shortlived nodes for making links, which would die without contributing much to data transfer after consuming important energy. Thus increasing energy efficiency of the whole network. Another important contribution of using energy- efficient nodes is, that solely with the use of different Ethresh values for different conditional (simulation time and number of nodes) or performance parameters, the network could be improved easily [21].

\section{Stability of the node}

Stability of a route at a given time can be measured by the stability of the nodes constituting the route for that given time period. The current protocol SLIMMER-SN aims to address the node stability in a network by taking the following factors:

a. Transmission range: Transmission Range (Rtx) represents the range within which a packet is successfully received if there is no interference from other radios. As transmission range decreases, it becomes difficult for nodes to reach receivers without more hops, while in case of high transmission range, requirement of more energy and increased interference deteriorates performance. So to get good performance an optimized value of transmission range should be chosen, we call it TRopt in the current study.

b. Node density: Node density in this study is taken as the number of neighbouring nodes. A low node density increases the unavailable intermediate routing nodes, whereas a high node density causes congestion. Also for a short transmission range,the increase in node density increases the performance, as increased number of hops helps a data packet to reach its destination. So an optimized value of node density needed to be chosen to get better performance; we call it NDopt in the current study.

c. Node velocity: An important factor causing frequent link breaks is high mobility of nodes, as it causes the node to move out of the sender's transmission range quickly, making the link 
unstable and prone to break. In case of nodes moving with a very small velocity delay increased, so to get good performance an optimized value of node velocity needed to be chosen, we call it $N$ Vopt in the current study.

In the current study, the effects of these three parameters were considered for defining the stability of a node by a metric SN.

d. Defining $S N$

Assumptions made while calculating SN:-

1. Each node can find its transmission range (TR), neighbour density (ND) and node velocity (NV).

2. The values of TRopt, NDopt and NVopt found after initial set of simulations, were used later in the studies.

Stability for a node $u$ at a time $t$ is given as $\mathrm{SN}_{\mathrm{u}}$ defined as a weighted function of $\mathrm{w} 1, \mathrm{w} 2$, and w3 such that

$$
\begin{aligned}
& \mathrm{SN}_{\mathrm{u}}=\mathrm{w} 1+\mathrm{w} 2+\mathrm{w} 3 \text { when TR= TRopt } \\
& \text { and } \\
& \mathrm{SN}_{\mathrm{u}}=(\mathrm{w} 1 \mathrm{x} \mathrm{w} 2)+\mathrm{w} 3 \text { in all other cases }
\end{aligned}
$$

Where $\mathrm{w} 1, \mathrm{w} 2$ and $\mathrm{w} 3$ are positive variable weight factors associated with transmission range (TR), node density (ND) and node velocity (NV) for a node $u$ at any time $t$.

In this study the values of w1, w2 and w3 vary as follows:

a. $\quad \mathrm{w} 1=\mathrm{w} 2=\mathrm{w} 3=1$ when $\mathrm{TR}=\mathrm{TRopt}$

b. $\quad \mathrm{w} 1=1$ if $\mathrm{TR}>=$ TRopt and -1 otherwise

c. $\quad \mathrm{w} 2=1$ if $\mathrm{ND}<\mathrm{NDopt}$ and -1 otherwise

d. $\quad \mathrm{w} 3=1$ if $\mathrm{NV}<=$ NVopt and 0 otherwise

The values for $\mathrm{w} 1, \mathrm{w} 2$ and $\mathrm{w} 3$ were taken only as a representative value to show the effects of each respective parameter .

\section{E. Cost function}

The problem of selecting the most efficient route in a network depends on the energy efficiency and stability requirements of the network. However these two parameters cannot be maximised together in most cases, hence a trade-off needs to be decided upon. In case of energy deficient but comparatively stable network, it is essential to select energy efficient nodes to create route, while on the other hand in case of highly dynamic environment with energy efficient nodes, selection of stable nodes should be favoured.Another application would use available nodes, energy efficient and/or stable, to create routes. Thus a weighted cost function $\mathrm{CF}$ is defined in equation 5 :

$$
\mathrm{CFj}=\alpha .(1 / \mathrm{Ei})+\beta .(1 / \mathrm{SNi}) \text { where } 1<=\mathrm{i}<=\mathrm{n}
$$

Where $\alpha$ is a weight associated with the reciprocal of energy efficiency of the node, Ei (residual energy of the node) and $\beta$ is the weight associated with reciprocal of stability of the node, SNi (Stability node factor). Ei and SNi for a node i are calculated according to equation (1) and equations (3 and 4) respectively. These are stored as fields in a control packet stored as multicast announcement (MA) in the study.

Reciprocals have been used so that the objective functions of the proposed mathematical model could be minimized.The optimal solution of the model introduced above is Pareto optimal and gives an idea about the shape of the Pareto surface and trade off among the various 
objectives [11].The user should choose appropriate values for the parameters $\alpha$ and $\beta$. In applications where energy efficiency is important or only energy efficient nodes are available $\alpha>>\beta$, whereas for applications where maintaining links and reducing delay are important and energy efficient nodes are not available, $\beta>>$.

\section{Stable Links In Multicast Mesh for Energy efficient Routing using Stable Nodes (SLIMMER-SN)}

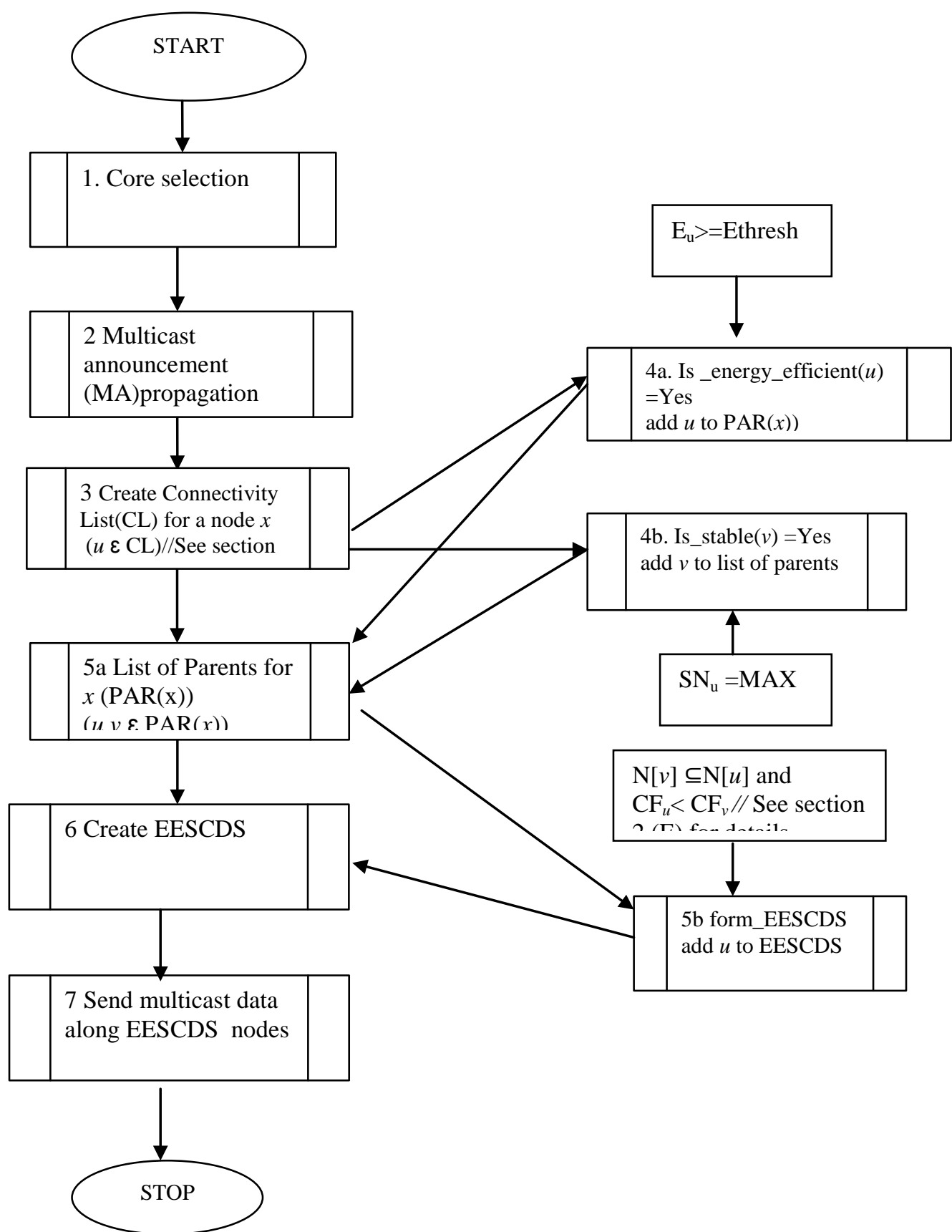

Fig. 1. Block diagram for functionality of SLIMMER-SN 
The flowchart in Fig. 1 gives the functionality of the proposed protocol The algorithm includes the following blocks or predefined processes:(i)Is _energy_efficient $(u)$ (ii)Is_stable(v) and (iii) form_EESCDS

The first receiver becomes the core and every receiver connects to the elected core, by using its address or the core id, by shortest paths.In case of more than one receiver, the one with the lower id is chosen as a node. The need of unicast messages is completely eliminated as SLIMMER-SN uses a single multicast announcement (MA) message to create and maintain mesh, election of cores and selection of routes. In PUMA the node with minimum hop count to the core is selected as the parent, or the preferred neighbour to reach the core. However these links may die out when nodes constituting them die out, due to lack of energy causing frequent link breaks. Also in case of PUMA, once a multicast data reaches a mesh member, it is flooded along the whole mesh causing redundancy, congestion, energy loss and link breaks.

Thus SLIMMER-SN improves upon its predecessors in:

1. Parent selection: In case of availability of more than one node at shortest distance, the energy of a node and its stability together with hop count from the core, are used to select parents.

2. Data transmission: A data packet is not flooded in the mesh, but sent across a set of forwarding nodes, this avoids the overheads incurred during flooding. Further the forwarding nodes are selected on account of (1) energy efficiency and (2) node stability. This leads to stable and energy efficient route selection.

The activities of SLIMMER-SN can be summarised as: The core sends MA, to create mesh and as the MA propagates a connectivity list is created at each node which stores data from all recent MA it receives. Out of all entries in the list, a node selects nodes at the shortest distance which are energy efficient and/or stable, as its parents depending on network conditions.The data packets are not flooded across the mesh but the protocol selects a set of forwarding nodes, for controlled data transmission.

\subsection{Network Model}

The network model can be given as an undirected graph $G=(V, E)$ where $V$ is the set of vertices and $\mathrm{E}$ is the set of bidirectional edges. Since SLIMMER-SN uses a structure based on a set of forwarding nodes, we define a few theoretical concepts before their usage further in the paper.

Definition1. Given a graph $G$, Dominating Set (DS) is a subset $D \subseteq \mathrm{V}$, such that for $V$, such that for every vertex $v \varepsilon V$, either $v \varepsilon D$, or $v$ has a neighbour in $D$.

Definition2. Connected Dominating Set (CDS): Given a graph G, a Connected Dominating Set (CDS) is a subset $D \subseteq V$ which satisfies the following two conditions.

- $\quad D$ is a DS

- $\quad$ For every two nodes $u$ and $v$ in $D$ there exists a path between $u$ and $v$ such that all intermediate nodes belong to $D$

Definition3. Graph G has a set of nodes V, such that V=F U P, and F $=\{s\} U$ L U R where

a) $\mathrm{F}$ is set of forwarding nodes, which receive and propagate packets

b) $\mathrm{P}$ a set of passive nodes, which only receive packets do not propagate them further.

c) L is a set of link nodes lying on the path between source and destination.

d) $s$ is the source and $\mathrm{R}$ is the set of receivers.

$\mathrm{F}$ and $\mathrm{P}$ satisfy the following conditions:

e) All nodes in $\mathrm{F}$ and $\mathrm{P}$ receive packets. 
f) Nodes in F propagate packets.

g) $\forall u \in P$ has at least one forward node as its neighbour,

h) Every forward node is connected to the source node via a path consisting of forward nodes only.

Energy Efficient Stable CDS (EESCDS): Given a graph G (V, E, and R) where $\mathrm{f}$ is a function which assigns weight to all the nodes $\mathrm{f}: \mathrm{V} \rightarrow \mathrm{R}+$. In case of our study, the energy efficiency and stability of node are assigned weights. EESCDS ensures that a node with lower weight than neighbours must be a member of CDS, keeping the total cost factor minimized.

Theorem 2. For $\mathrm{v} \in \mathrm{V}, \mathrm{N}(\mathrm{v})$ denotes neighbour set, and $\mathrm{N}(\mathrm{v})=\{\mathrm{u} \mid(\mathrm{u}, \mathrm{v}) \in \mathrm{E}\}$ and $\mathrm{a}$ broadcasting ensures coverage, if every node receives the broadcast packets; that is, $\mathrm{V}-\mathrm{F} \subseteq$ $N(F)$, where $N(F)=U_{v \varepsilon F} N(v)$, and $v$ is a forwarding node and $N(F)$ is a set of union of all neighbours of $\mathrm{v}$.

Lemma1: EESCDS ensures coverage

Proof: The proof is presented as a contradiction so let us assume that the CDS created does not provide full coverage thus there are nodes which do not receive the packet as they have no neighbour in the forwarding set.; we call such nodes unvisited node or UV.

For any given network represented as a graph $\mathrm{G}(\mathrm{V}, \mathrm{E})$ with $s$ as the source node let FN be the set of forwarding nodes, which both receive as well as carry the packets. Also, let PN be the set of passive nodes, which only received the packets, but did not carry them to UV. Apparently $\mathrm{PN} \neq \varnothing$, because if there were no passive nodes, then the graph would not be connected and UV nodes would be separated from source node. Thus UV $=\mathrm{V}-\mathrm{FN}-\mathrm{PN}$ and $\mathrm{UV} \neq \varnothing$ [as per assumption].So as in Fig. 2, we have an unvisited node $x$ which did not receive a packet, because the neighbouring node $w$ with the lowest CF value, is a passive node. Also by definition since each passive node should have at least one forwarding node as a neighbour, so let there be a node $y$ as a neighbouring forward node to $w ; y \varepsilon \mathrm{N}(w)$ and $y \varepsilon \mathrm{FN}$. According to our definition of EESCDS, a forwarding node should have the minimum CF in the neighbourhood so CFy should be less than CFw. However, as per our assumption w has the least CF in the neighbourhood; this contradicts our choice of $y$ as a forwarding node for energy efficient stable CDS. Hence, our assumption is wrong and $w$ should be a member of FN. Thus EESCDS implies, that there is no unvisited node $x$ or $\mathrm{U}=\mathrm{V}-\mathrm{F}-\mathrm{N}(\mathrm{F})$, is empty. Explanatory diagram for the proof, is given in Fig. 2. Let f's represent forwarding nodes.

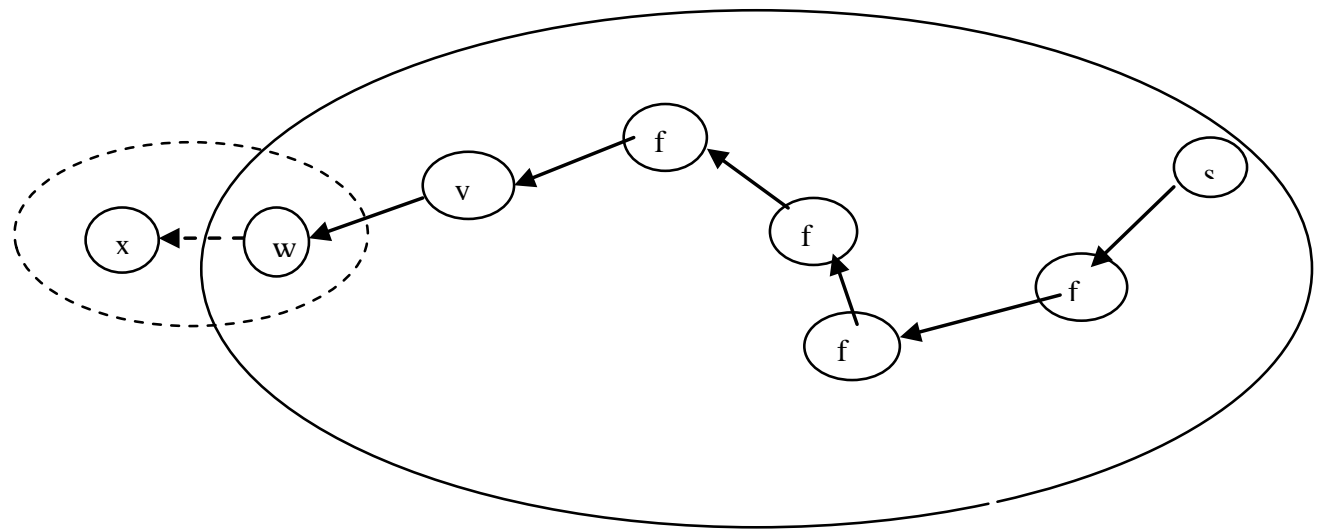

Fig. 2. EECDS ensures coverage 


\subsection{Multicast Announcement}

SLIMMER-SN uses a multicast announcement (MA) for creating and maintaining mesh, selection of core and route. The structure of MA in SLIMMER-SN is given in Fig. 3.

\begin{tabular}{|c|c|c|}
\hline Mesh member & SN & Energy \\
\hline \multicolumn{3}{|c|}{ Core id } \\
\hline \multicolumn{3}{|c|}{ Group id } \\
\hline \multicolumn{3}{|c|}{ seq number } \\
\hline \multicolumn{3}{|c|}{ parent } \\
\hline \multicolumn{3}{|c|}{ Distance to the core } \\
\hline
\end{tabular}

Fig. 3. Structure Multicast Announcement SLIMMER-SN

The fields specified by a MA are as follows:

$>$ Core ID: core ID in the best multicast announcement

Group ID: group ID in the best multicast announcement

Sequence number: sequence number in the best multicast announcement

$>$ Distance to core: One plus the distance to core in the best multicast announcement

$>$ Parent: The neighbour from which it received the best multicast announcement

$>$ Mesh member status: whether a particular node is a mesh member

$>$ SN: SN value of the node

$>\quad$ Energy: remaining energy value of node

Mesh member status: A node sets its status to true if it is a mesh member when

$>\quad$ It is a receiver

$>$ Non-receivers consider themselves mesh-members if they have at least one mesh child in their connectivity list. A neighbour in the connectivity list is a mesh child if :

(1) its mesh member flag is set, (2) the distance to core of the neighbour is larger than the node's own distance to core, and (3) the multicast announcement corresponding to this entry was received in within a time period equal to two multicast announcement intervals

First two conditions ensure that if a node has a mesh child then it lies on a shortest path from a receiver to the core and should therefore be a mesh member. Condition 3 is used to ensure that a neighbour is still in the neighbourhood.

Multicast announcement (MA) propagation

A node that considers itself to be the core of a group transmits multicast announcements periodically for that group. As the multicast announcement travels through the network, it establishes a connectivity list(CL) at every node which stores the data received from all the multicast announcements from its neighbours, with the time when it was received. Successive MAs from the same core have a higher sequence number; the node keeps only one most recent entry in its connectivity list from a particular neighbour. It then generates its own multicast announcement, based on the best entry in the connectivity list. Nodes which are neither energy efficient nor with maximum stability node index, SN will not be considered for parenthood After selecting the best multicast announcement, the node generates the fields of its own multicast announcement. In case of core the parent field is kept as invalid address and distance to core as zero. Fig. 4 gives the propagation of MAs from neighbouring nodes to a node 5 .

Say the connectivity list for node 5 has entries for its neighbours 4, 8 and 11 respectively given in Table 1. While all 4, 8 and 11are at the same distance from the core, 4 and 8 are energy efficient and node 11 has highest SN, value so all are prospective candidates for parents 
depending on the prevailing network conditions.Table 1 gives connectivity list for the node 5.

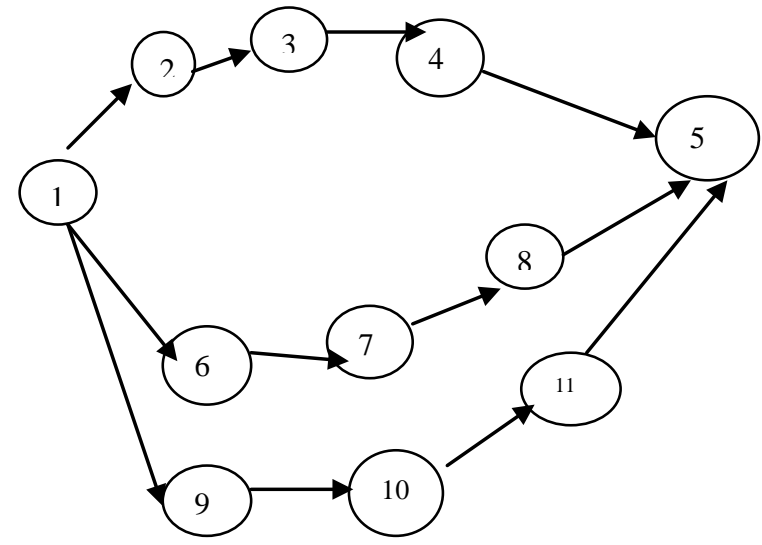

Fig. 4. Propagation of Multicast Announcement

Table 1. Connectivity list of node $5($ Core ID $=1$, Group ID = 224.0.0.1, Seq. No. $=79)$

\begin{tabular}{|c|c|c|c|c|c|}
\hline \multirow[t]{2}{*}{ Neighbor } & \multicolumn{4}{|c|}{ Multicast announcement } & \multirow{2}{*}{$\begin{array}{l}\text { Time } \\
\text { (ms) }\end{array}$} \\
\hline & Distance to core & Parent & Energy status & SN & \\
\hline 4 & 3 & 3 & $99 \%$ & 2 & 12552 \\
\hline 8 & 3 & 8 & $88 \%$ & 1 & 12180 \\
\hline 11 & 3 & 10 & $20 \%$ & 3 & 12182 \\
\hline
\end{tabular}

After waiting for some time, to collect updated MAs from its neighbours node 5, makes its own MA. There can be more than 1 parent for a node and in that case MA shall propagate through all parents.

\subsection{Mesh Establishment and Maintenance}

A mesh is established by all shortest path routes between a source and receiver pair. MA is used to create and maintain this mesh which excludes energy deficient and unstable nodes. After receiving of MA each node updates its connectivity list and selects the best entry or the parent(PAR)

\subsection{Multicast data packets}

In most of multicast mesh protocols, when the data packet reaches a mesh member, it is flooded within the mesh, and nodes maintain a packet ID cache to drop duplicate data packets, while in SLIMMER-SN cost function is used to decide the forwarding set, which would propagate data rather than flooding it throughout all the routes in the mesh. This cost function tradeoff between parameters associated with energy efficiency and stability. So data transmission in SLIMMER-SN follows the following steps:

- When $x$ a non mesh member node receives multicast data packet, it unicasts the packet to a mesh member according to conditions given in 3.2. 
- When $x$ is a mesh member, it determines its forwarding set $\mathrm{F}$ and transmits the data packet to members of $\mathrm{F}$.

- $\quad$ A non member of forwarding set doesn’t retransmit the packet further but receives it.

\section{Experimental Results and Analysis}

To demonstrate the effectiveness of SLIMMER-SN, two sets of comprehensive simulations were performed on NS-2.35.

1. The first set of simulations was performed to (1) measure the effect of transmission range (ii) node density and (iii) node velocity on performance metrics and (2) find the values of TRopt, NDopt and NVopt.

2. The second set of simulations was performed to compare the performance of PUMA, SLIMMER and SLIMMER-SN under varying number of nodes.The results of first set of simulations have been used for the second set of simulations. Since performance improves with increased stability, the performance metrics PDR, Throughput, Delay and Average energy consumed were taken as a measure of stability for this study.PDR is the ratio of received packets to sent packets (including retransmitted packets). Throughput is the number of packets received per unit time. Delay gives the time latency between a packet sent to the packet received. Average energy consumed is a measure of energy consumed by each node. Assumptions:

- In this study maximum transmission power used is $(\mathrm{Pt})=0.28183815 \mathrm{~W}$, that can cover a maximum fixed transmission range of $250 \mathrm{~m}$.

- $\quad$ The study further assumes that the transmission range and the carrier sensing range are identical, a packet if sensed, can be received [23].

- $\quad$ Transmission ranges were set by taking different values of receiving threshold (Rx) by modifying ns/indep-utils/propagation/ threshold.cc provided with the ns source files.

- $\quad$ All nodes are assumed to be energy efficient for Ethresh $=30 \%$ of initial energy [24] (for this study Ethresh=.3J).Initial energy in this study is $1 \mathrm{~J}$.

\subsection{Experiment 1 to demonstrate relation between TR, ND and NV}

Experiment 1 was carried out to find the effect of parameters: transmission range,node density and node velocity on performance of the network.Case 1 discusses the effects of only TR on performance whereas, Case2 studies the dual effect of TR and Node density. For the experiment purposes the total number of nodes is taken as the node density, as the node density will vary directly with number of nodes Case3 is a study about the dual effects of TR and Node Velocity.Simulation scenario for the first set of experiments given in Table 2.

Table 2. Simulation scenario for first set of simulations

\begin{tabular}{|l|l|l|}
\hline \multicolumn{1}{|c|}{ Parameter } & \multicolumn{1}{|c|}{ Value } & units \\
\hline Total Nodes & $100,200,300,400,500,600,700,800,900,1000$ & - \\
\hline Transmission range & $50,75,100,125,150,225,250$ & $\mathrm{~m}$ \\
\hline Node velocity & $5,10,15,20,25,30$ & $\mathrm{~m} / \mathrm{s}$ \\
\hline Ethresh & 0.3 & Joule \\
\hline Simulation Area & $1000 \times 1000$ & $\mathrm{M}^{2}$ \\
\hline Simulation time & 100 & $\mathrm{sec}$ \\
\hline
\end{tabular}


Case1. Results for effect of transmission range on (a) PDR (b) Throughput and (c) Delay are given in Figs. 5-7 respectively.

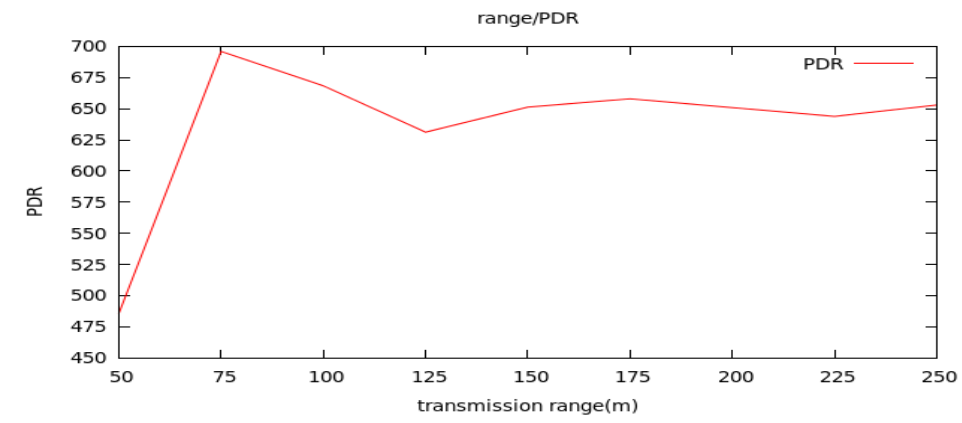

Fig. 5. Effect of Transmission range(TR) on packet delivery ratio(PDR)

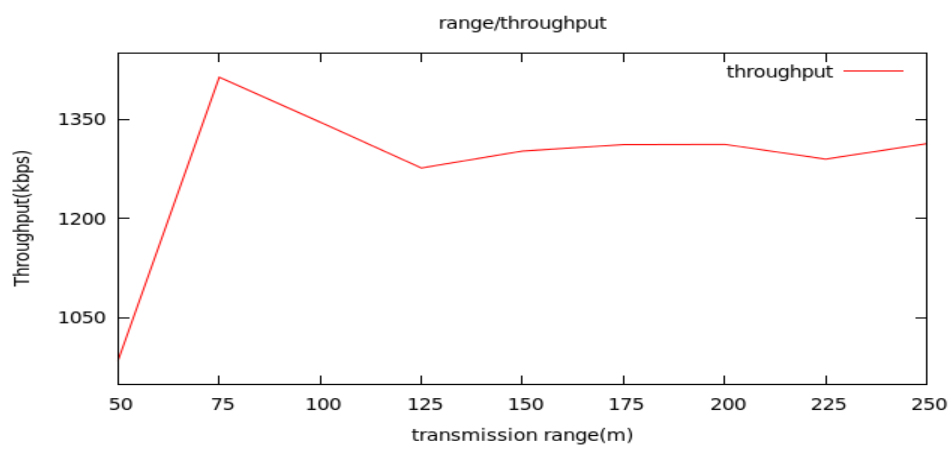

Fig. 6. Effect of Transmission range (TR) on throughput

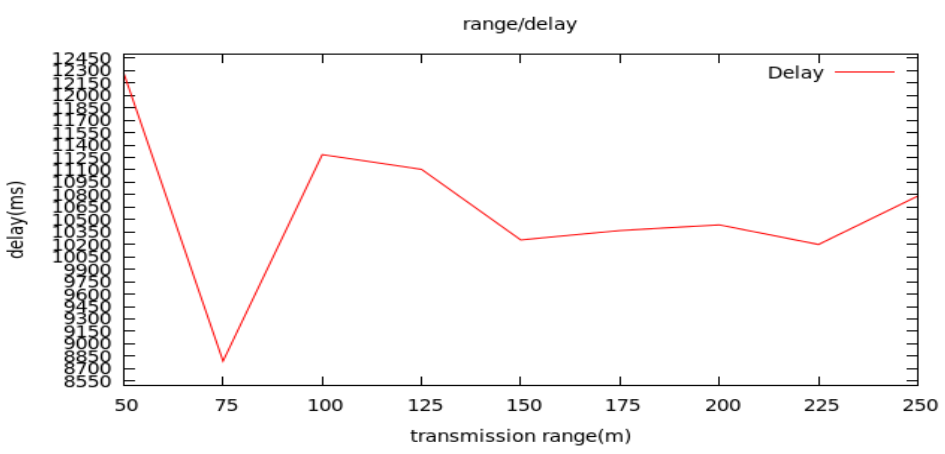

Fig. 7. Effect of Transmission range (TR) on Delay

It could be seen that when the transmission range is very small $(50 \mathrm{~m})$ the nodes are practically isolated with small communication overlap between sender and receiver, as a result the delay is more and PDR and throughput less. When the range increases $(75 \mathrm{~m})$ and the receiver and sender communicate directly, the values of all parameters improve up to a certain level.Large transmission range $(>75 \mathrm{~m})$ causes more interference, more energy consumption which results in nodes dying out. Larger values of TR do not add to the performance as the nodes do not add to efficient routes but just add to congestion.An optimum level of transmission range which gives best performance in our case, we term it as TRopt, was found at $75 \mathrm{~m}$. 
Case2. Results and discussion for the dual effect of transmission range and Node density. The effects of node density (ranging from 100-1000 nodes) were observed for four different transmission ranges:

- When transmission range was very low $(50 \mathrm{~m})$ for $\mathrm{Rx}=7.69113 \mathrm{e}-08$

- When transmission range was optimum or TRopt $(75 \mathrm{~m})$ for $\mathrm{Rx}=3.417817 \mathrm{e}-08$ (as per previous result).

- When transmission range was high $(150 \mathrm{~m})$ for $\mathrm{Rx}=2.81 \mathrm{e}-09$.

- When transmission range was highest $(250 \mathrm{~m})$ for $\mathrm{Rx}=3.65262 \mathrm{e}-10$ (for transmission power $=.28 w$ ). The results are shown in Figs. 8- 10.

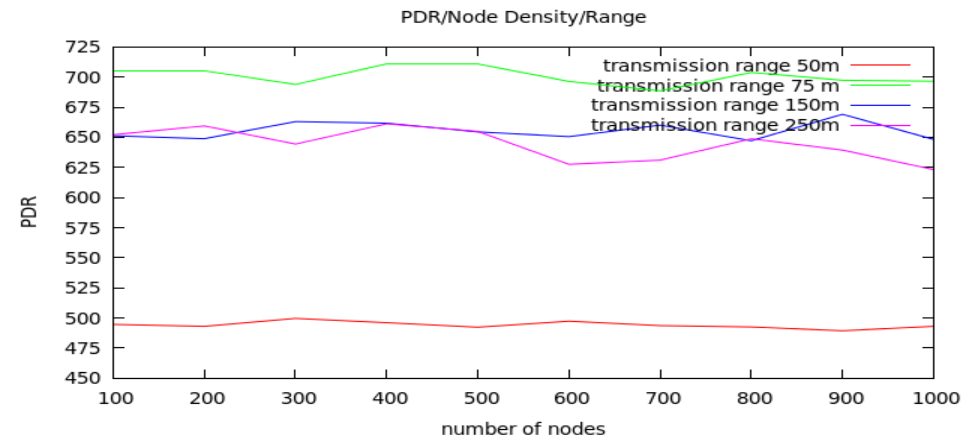

Fig. 8. Effect of TR and Node density on PDR

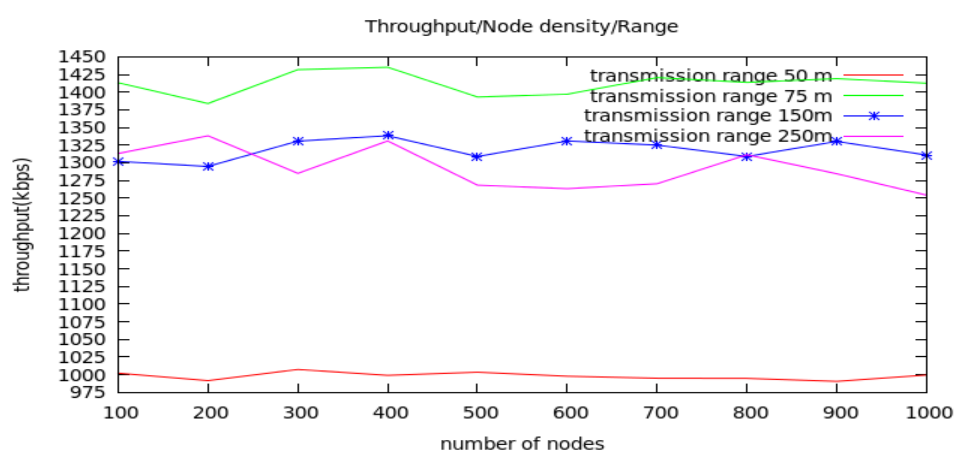

Fig. 9. Effect of TR and Node density on throughput

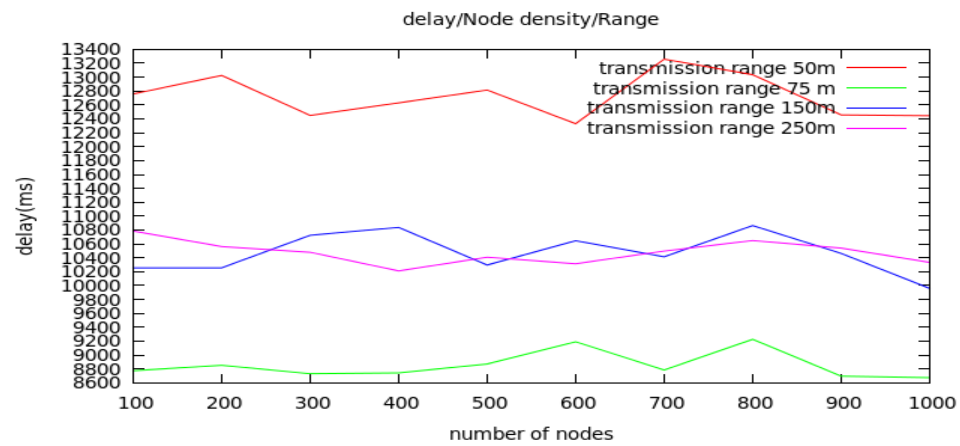

Fig. 10. Effect of TR and Node density on delay 
For very low transmission range $(50 \mathrm{~m})$ the increase in the number of nodes, increases the throughput, as more relay nodes are available, however, it does not affect the PDR, the reason could be that receivers lie far on the periphery and all sent packets cannot be received. Also for low transmission range more hops are needed till destination, this increased hop count increase the delay.For an optimum range (75 m) PDR and throughput increase and delay decreases as more intermediate nodes are available and nodes can communicate more freely. However, after 400 nodes further increase in the number causes congestion and decrease in performance. With a further increase in transmission range $(150 \mathrm{~m})$ the PDR and throughput value maintain good values. When the transmission range is increased to the highest limit of the transmission power (250 m) PDR and throughput decrease as control packet traffic, interference and energy consumption increase. Also, as the number of nodes increase availability of more relay nodes, cause a decrease in delay, but a further increase of around 700 nodes cause link breaks, due to congestion, and delay increases.

Case 3. Results and discussion for the dual effect of transmission range and Node velocity are given in Figs. 11-13 as in previous experiment.

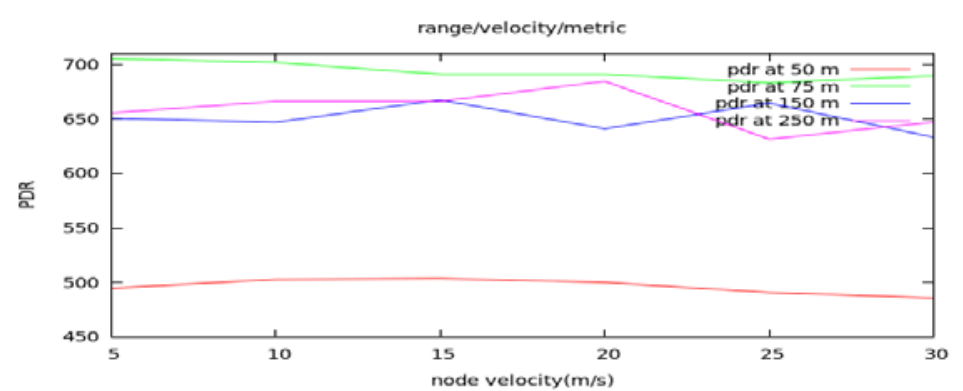

Fig. 11. Effect of TR and Node velocity on PDR

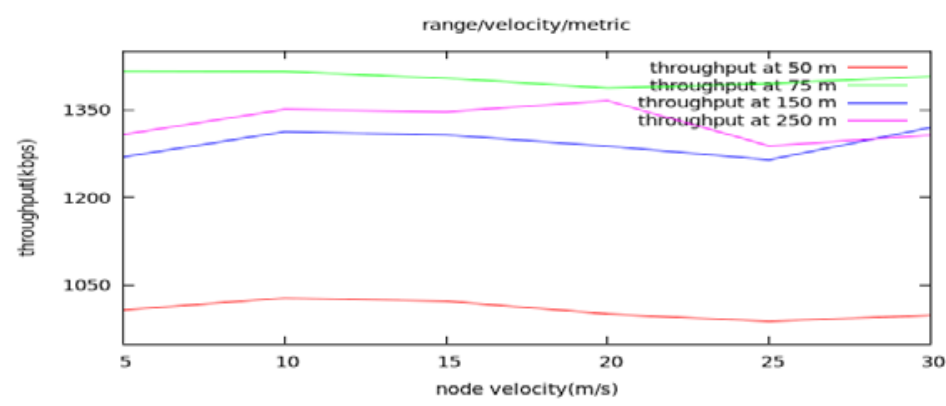

Fig. 12. Effect of TR and Node velocity on throughput

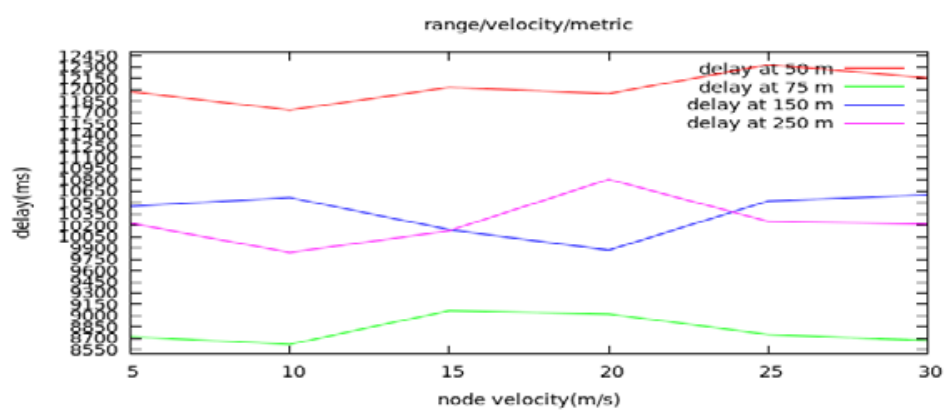

Fig. 13. Effect of TR and Node velocity on delay 
For a very small transmission range (50 m) PDR and Throughput are stable, marginally dipping at high velocity. The reason could be that higher mobility, although increases chances of more nodes coming in contact, but the nodes do so with low communication interval.

At a long transmission range $(150 \mathrm{~m})$ PDR follows the irregular pattern while throughput follows the same pattern as in case of small transmission range, as availability of nodes varies. For ranges at the highest limit of transmission (250 $\mathrm{m}$ in this study) throughput and PDR increases with increase in node velocity ( up to $20 \mathrm{~m} / \mathrm{s}$ ) as nodes travel faster to reach destination.The availability of faster moving relay nodes decreases the delay.A further increase in velocity results in link breaks as communication overlaps between nodes decreases.

At an optimized range (75 m): All performance parameters give good values and remain stable even with an increase in velocity. After around $10 \mathrm{~m} / \mathrm{s}$ the performance starts falling for this TR so we take NVopt as $10 \mathrm{~m} / \mathrm{s}$ for this transmission range in this study.

As can be observed the results justify the general observations.

The results after experiment 1 (i) measured the effect of all the selected parameters energy, transmission range, node density and node velocity of performance metrics effected by link stability and (ii) derived the optimal values of all the parameters .

\subsection{Experiment 2 to compare the performance of PUMA, SLIMMER and SLIMMER-SN}

The performance of PUMA, SLIMMER and SLIMMER-SN was compared for packet delivery ratio.throughput, delay and average energy consumed.The comparison was done for two cases, case 1 for varying number of nodes and case 2 for varying simulation time.

The simulation scenario for experiment 2 is given under Table 3 .

Case 1. Results for PDR, throughput, delay and average energy consumed for increasing number of nodes are given in Figs. 14-17.

Assumptions

1. The set of simulations in experiment 2, use the results obtained in the first.

2. For SLIMMER and SLIMMER-SN Ethresh=30\% of initial energy [24].

3. $\quad \mathrm{TR}=\mathrm{TRopt}, \mathrm{NV}=\mathrm{NVopt}$ [as result of experiment 1 ].

4. For the present study $\alpha=\beta=0.5$ was taken (equal weight to energy efficiency and stability ).

Table 3. simulation scenario for second set of simulations

\begin{tabular}{|l|l|c|}
\hline Parameter & Value & units \\
\hline Total Nodes & $\begin{array}{l}\text { 1. 100, 200,300,400,500,600,700,800,900,1000 } \\
\text { 2. 100 }\end{array}$ & - \\
\hline Routing protocols & PUMA,SLIMMER and SLIMMER-SN & $\mathrm{m}$ \\
\hline Transmission range & $75(\mathrm{TRopt})$ & $\mathrm{m} / \mathrm{s}$ \\
\hline Node velocity & 10 (NVopt) & Joule \\
\hline Initial energy & 1 & Joule \\
\hline Ethresh & 0.3 & $\mathrm{~m}^{2}$ \\
\hline Simulation Area & $1000 \times 1000$ & secs \\
\hline Simulation time & 1.100 & \\
\hline
\end{tabular}




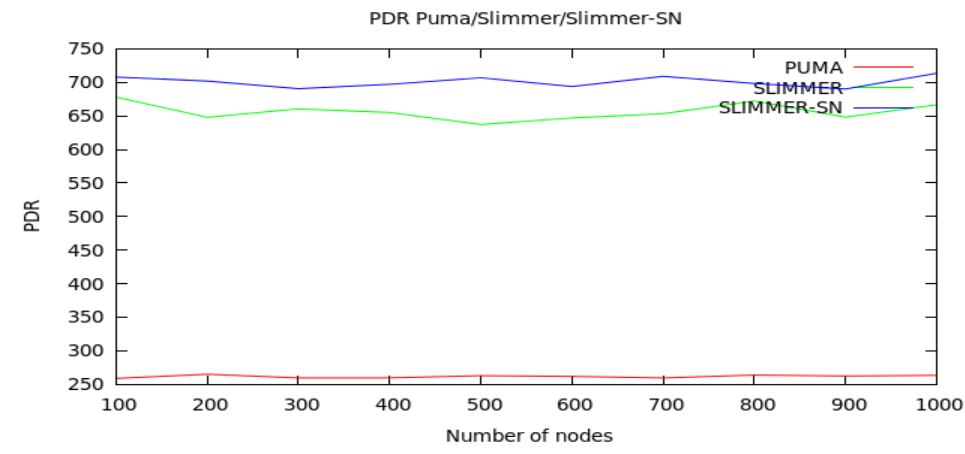

Fig. 14. Comparative PDR for PUMA, SLIMMER and SLIMMER-SN with number of nodes

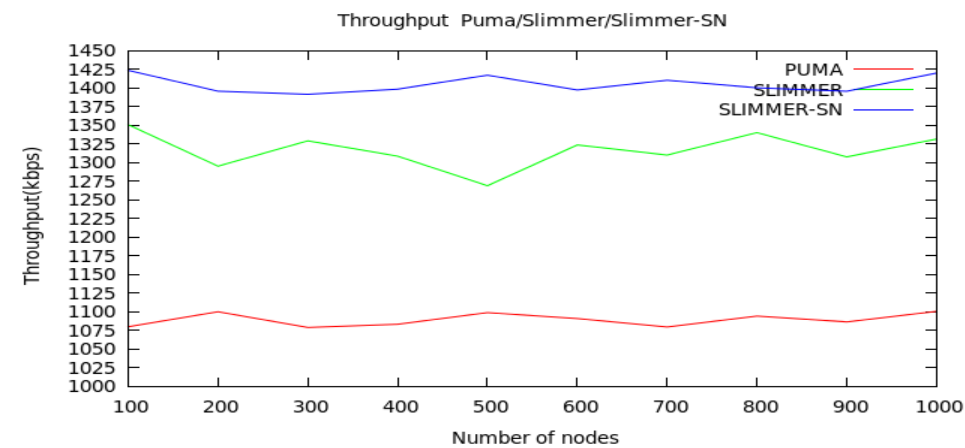

Fig. 15. Comparative throughput for PUMA, SLIMMER and SLIMMER-SN with number of nodes

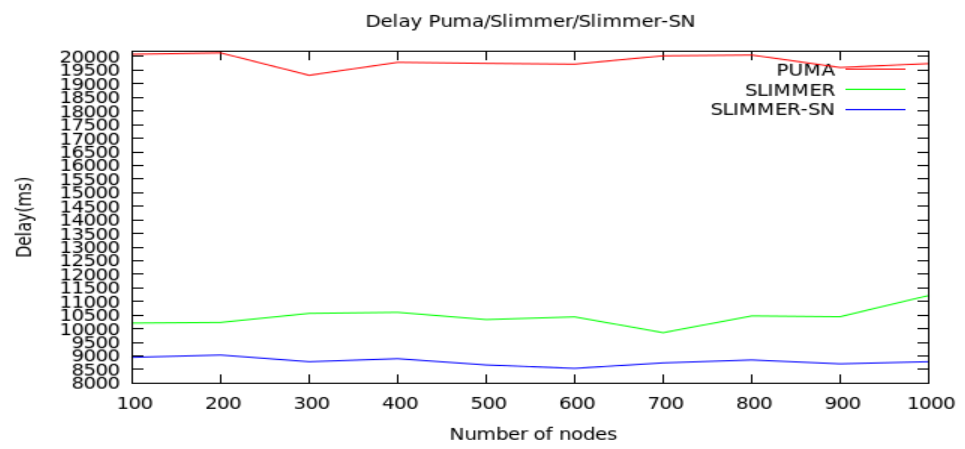

Fig. 16. Comparative delay for PUMA, SLIMMER and SLIMMER-SN with number of nodes

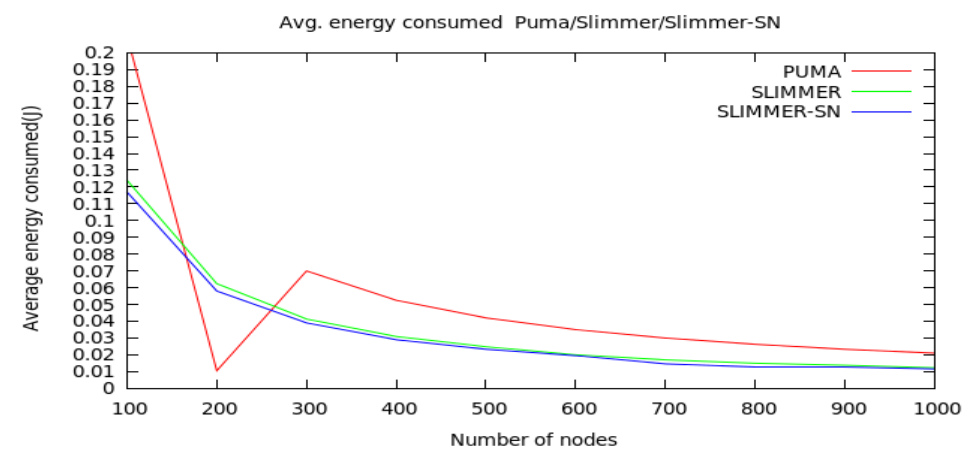

Fig. 17. Comparative avg. energy for PUMA, SLIMMER and SLIMMER-SN with no. of nodes 
SLIMMER and SLIMMER-SN both use energy efficient nodes so their average energy consumed is same but better than PUMA.With increase in the number of nodes, congestion increases, but for SLIMMER and SLIMMER-SN this also means more energy efficient nodes, thus the detrimental effect is taken care of.Delay increases in PUMA as links break and new links are established, in SLIMMER for a high number of nodes around 800, congestion overpowers the requirement of energy efficiency as new nodes do not add to stability but increase traffic. SLIMMER-SN outperforms both as it decides dynamically and uses energy efficient or stable nodes whichever are available.

Case 2 : Results for PDR, throughput, delay and average energy consumed with increasing simulation time are given in Figs. 18-21.

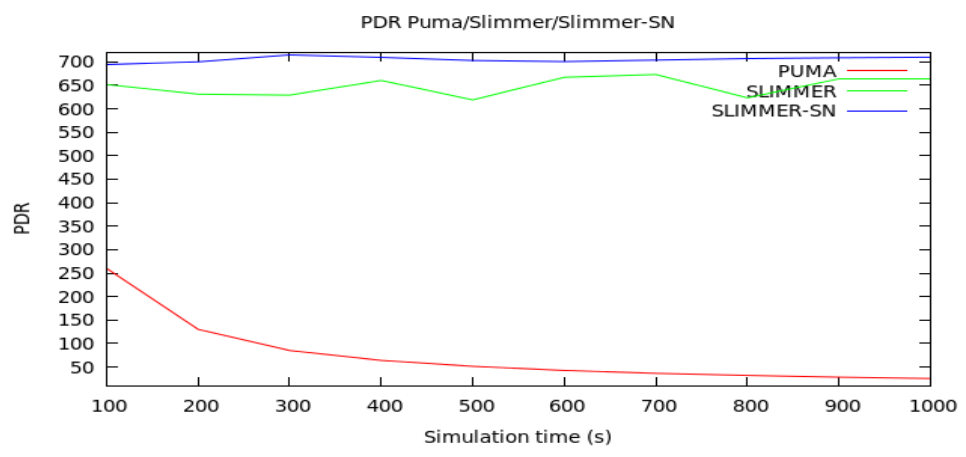

Fig. 18. Comparative PDR for PUMA, SLIMMER and SLIMMER-SN with simulation time

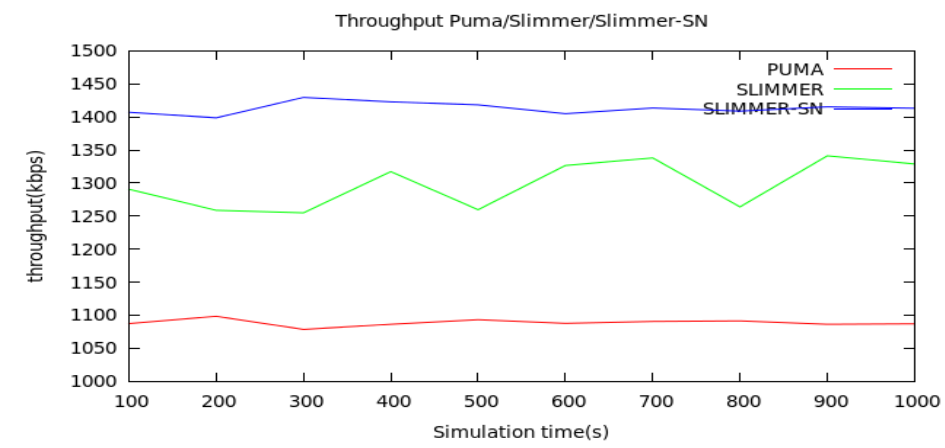

Fig. 19. Comparative throughput for PUMA, SLIMMER and SLIMMER-SN with simulation time

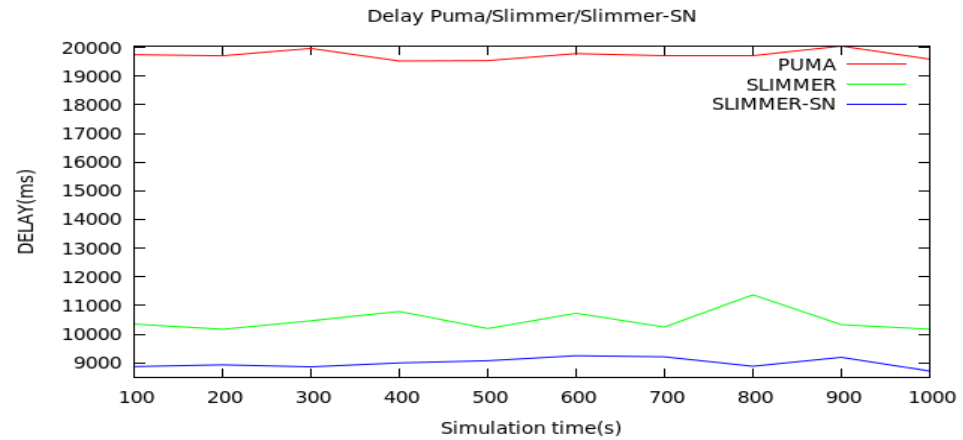

Fig. 20. Comparative delay for PUMA, SLIMMER and SLIMMER-SN with simulation time 


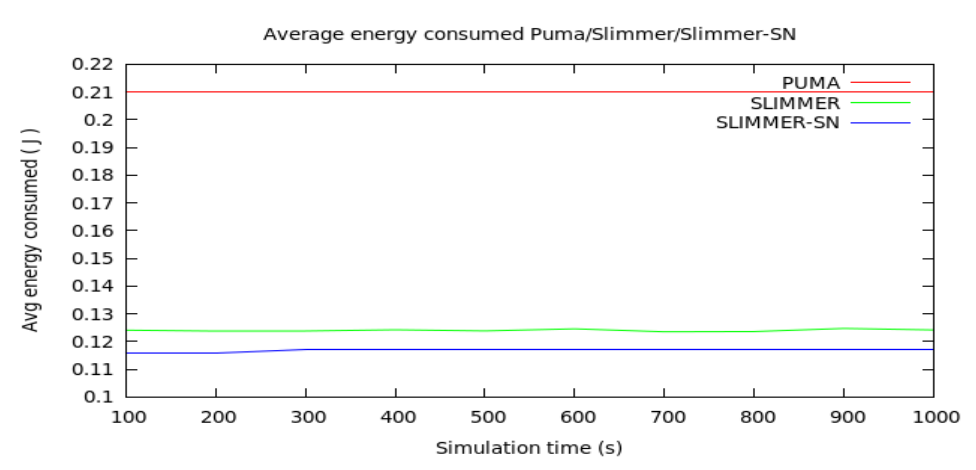

Fig. 21. Comparative avg. energy consumed for PUMA, SLIMMER,SLIMMER-SN with simulation time

For PUMA as nodes lose energy with time, availability of energy efficient nodes in PUMA decreases, so PDR decreases with more frequent link breaks. This causes irregularities in PDR for SLIMMER. However, energy efficient nodes in case of SLIMMER help the network in stabilising after retransmissions. In case of SLIMMER-SN, when/if energy efficient nodes are not available, the stable nodes are chosen to form links, and vice- versa; thus PDR and throughput in this case show considerable improvement over the other two protocols. In case of throughput for PUMA, the effect is not that pronounced as retransmission of packets do not affect the throughput. SLIMMER and SLIMMER-SN follows the same pattern as that for throughput. Delay and average energy show similar behaviour with varying time.

Table 4 given below gives consolidated results for both the cases.

Table 4. Consolidated outcomes

\begin{tabular}{|l|c|c|c|}
\hline & PUMA & SLIMMER & SLIMMER-SN \\
\hline PDR & Lowest & Good & Best \\
\hline Throughput & Lowest & Good & Best \\
\hline Delay & Highest & Low & Low \\
\hline Avg. Energy consumed & High & Low & . \\
\hline
\end{tabular}

\section{Conclusion \& future work}

MANETs face two important constraints of limited battery and frequent link breaks, both of which are taken care of in the proposed protocol. The proposed protocol SLIMMER-SN gives much better performance than both PUMA and SLIMMER, as nodes making mesh are energy efficient with maximum node stability. However the values of optimum transmission range, node density, node velocity, alpha and beta have been taken as per the results of our experiments and so these values may differ according to different network applications. The future work includes studying the effect of the speed at which the node drains the battery, on route stability and energy efficiency computation.Further, only some of the performance parameters have been compared in the current study, however there are various other parameters like packet size, jitter etc which also play very important role in determining efficiency of a protocol. Authors plan to compare and analyze some of the other performance parameters as a part of future work.An interesting area of research to analyze the failure situation of the protocol and applying the research to other areas. 


\section{References}

[1] J. Loo, J.L. Mauri and J.H. Ortiz, Mobile ad hoc networks: current status and future trends, CRC Press, Boca Raton, 2016. Article (CrossRef Link).

[2] L.Junhai,Y. Danxia, X. Liu and F. Mingyu, "A survey of multicast routing protocols for mobile ad-hoc network,” IEEE Commun. Surv. Tut, vol. 11(1), pp. 78-91, 2009. Article (CrossRef Link).

[3] C.Rajan and N.Shanthi, "Genetic based optimization for multicast routing algorithm for MANET,” Sadhana, vol. 40 (8), pp.2341-2352, 2015. Article (CrossRef Link).

[4] R.C. Biradar,S.S Manvi and M. Reddy, "Mesh based multicast routing in MANET: stable link based approach,” Int. J. Comput. Electr. Eng, vol. 2, pp.371-380, 2010. Article (CrossRef Link).

[5] A.Al-Hemyari,M. Ismail,R. Hassan and S. Saeed, "Improving link stability of multicast routing protocol in manets,” J. Theor. Appl. Inf. Technol,vol. 55(1), 2013.

[6] M. Gerharz, C. De Waal, M. Frank and P. Martini, "Link stability in mobile wireless ad hoc networks,” in Proc. of Local LCN 27th Annual IEEE Conference, pp. 30-39, 2002. Article (CrossRef Link).

[7] P.I. Basarkod and S.S. Manvi, “On-demand QoS and Stability Based Multicast Routing in Mobile Ad Hoc Networks,” J. Telecommun. Inform. Techno, vol. 3(98), 2014.

[8] M. Ali , F. Semchedine and A. Boukerram, "A link-state QoS routing protocol based on link stability for Mobile Ad hoc Network,” J. Netw. Comput. Appl. vol. 39, pp.117-125, 2014. Article (CrossRef Link).

[9] H. Noureddine, Q. Ni, G. Min and H.Al-Raweshidy, "A new link lifetime estimation method for greedy and contention-based routing in mobile ad hoc networks,” Telecomm. Systems, vol. 55(3), pp. 421-433, 2014. Article (CrossRef Link).

[10] N. Papanna, A.R.M. Reddy and M. Seetha, "EELAM: Energy Efficient Lifetime Aware Multicast Route Selection for Mobile Ad Hoc Networks,” Appl. Comput. and Inform, vol. 15(2), pp. 120-128, 2019. Article (CrossRef Link).

[11] F. De Rango, F. Guerriero and P.Fazio, "Link-stability and energy aware routing protocol in distributed wireless networks,” IEEE Trans. on Parallel and Distributed Processing, vol. 23 (4), pp.713-726, 2012. Article (CrossRef Link).

[12] V.K. Sharma and M. Kumar, "Adaptive Energy Efficient Load Distribution Using Fuzzy Approach,” Ad Hoc Sens. Wirel. Ne., vol. 39, 2017.

[13] S. Xiang and J. Yang, "Performance reliability evaluation for mobile ad hoc networks," Reliab. Eng. Sys. Safe. vol. 169, pp. 32-39, 2018. Article (CrossRef Link).

[14] R. Vaishampayan and J.J., "Efficient and robust multicast routing in mobile ad hoc networks," in Proc. of the 1st IEEE international conference on mobile ad-hoc and sensor systems (MASS), 2004. Article (CrossRef Link).

[15] K. Vyas, A. Khunteta and A. Chaturvedi, "SLIMMER: Stable Links in Multicast Mesh for Energy Efficient Routing," in Proc. of IEEE Int. Conf. on Recent Advances and Innovations in Eng.(ICRAIE), pp. 1-5, 2016. Article (CrossRef Link).

[16] E. Astier , A. Hafid and S. Aljahdali, "An efficient mesh based multicast routing protocol in mobile ad hoc networks,” Wireless Comm. and Mobile Comput., vol. 12(9),pp. 822-836, 2012. Article (CrossRef Link).

[17] R. Menchaca-Mendez, R. Vaishampayan, J.J. Garcia-Luna-Aceves and K. Obraczka, "DPUMA: a highly efficient multicast routing protocol for mobile ad hoc networks," in Proc. of Int. Conf. on Ad-Hoc Networks and Wireless Springer, Berlin, Heidelberg, pp. 178-191, 2005. Article (CrossRef Link).

[18] J. Wu, and F. Dai, “A generic broadcast protocol in ad hoc networks based on self-pruning," in Proc. of IEEE Int. Symp. on Parallel and Distributed Processing, vol. 8, 2003.

[19] F. Vazquez-Araujo, A. Dapena , M.J. Souto-Salorio and P.M. Castro, "Calculation of the Connected Dominating Set Considering Vertex Importance Metrics,” Entropy, vol. 20(2), pp. 87, 2018. Article (CrossRef Link).

[20] H. Zhang and Z.P. Jiang, "Mobility Sensitive Broadcast Algorithms in Highly Mobile Ad Hoc Networks,” Ad Hoc Sens. Wirel. Ne.,vol. 3, pp. 171-196, 2007. Article (CrossRef Link). 
[21] K. Vyas, A. Khunteta and A. Chaturvedi, "Catching energy efficient stable nodes in Ad-hoc Networks,” Int. J. of Appl. Eng. (IJAER), vol. 13 (05), pp.2490-2498, 2018.

Article (CrossRef Link).

[22] H. Lim and C. Kim, "Flooding in wireless ad hoc networks," Comput. Commun., Elsevier, 24, pp. 353-363, 2001. Article (CrossRef Link).

[23] J. Marchang, B. Ghita B and D.Lancaster, "Location based transmission using a neighbor aware-cross layer MAC for adhoc networks,” in Proc. of WWIC 2016, LNCS 9674, pp. 15-27, 2016. Article (CrossRef Link).

[24] Y. Park and S. Lee, "A routing protocol for extend network lifetime through the residual battery and link stability in MANET," in Proc. of Applied computing conference (ACC '08), Istanbul, Turkey, pp. 199-204, 2008. Article (CrossRef Link).

[25] A. Sufian, A. Banerjee, and P. Dutta,"Energy and Velocity Based Tree Multicast Routing in Mobile Ad-Hoc Networks," Wireless Personal Communications, vol.107(4), pp.2191-2209, 2019. Article (CrossRef Link).

[26] A.Tavizi and A. Ghaffari, "Tree-based reliable and energy-aware multicast routing protocol for mobile ad hoc networks," The Journal of Supercomputing , vol.74, no. 11, pp. 6310-633, 2018. Article (CrossRef Link).

[27] L. Femila and M. M. Beno, "Optimizing Transmission Power and Energy Efficient Routing Protocol in MANETs,” Wireless Personal Communications, vol.106(3), pp. 1041-1056, 2019. Article (CrossRef Link).

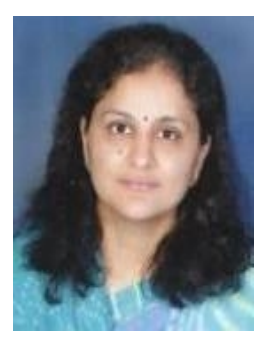

Kapila Vyas is currently a research scholar at Poornima University Jaipur. Her research interests include ad hoc networks, routing protocols, simulation, and network performance evaluation

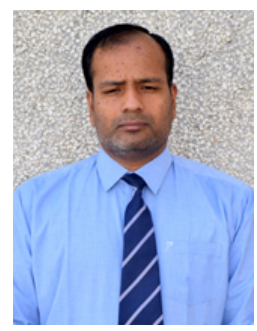

Dr. Ajay Khuteta has 16 years of teaching and research experience. He completed his doctorate on Distributed Systems. He has guided $34 \mathrm{M}$. Tech and $01 \mathrm{Ph}$. D students in the field of Distributed Algorithms, Information Security, Cloud Computing, Big Data, and Image Processing. He has published 54 research papers in national/International Journals and conferences. He is Member of IEEE, ISTE and IEI

Email : khutetaajay@poornima.org

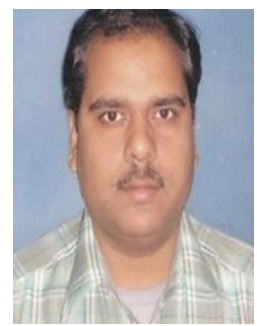

Dr. Amit Chaturvedi obtained the MCA degree in 2001 and Ph.D. degree in Mar, 2012. He is presently teaching in the Govt. Engineering College, Ajmer. He has 17 years long PG teaching experience. Five doctorate degrees are awarded under his supervision. He has published around 72 research papers in national/international Journals and conference. Three govt funded projects are successfully completed by him and obtained the grants from DST, Rajasthan and AICTE, New Delhi. He has written three text books in the computer science subjects. Presently he is working on the subjects of cloud computing and multicast communication in adhoc networks

Email : amit0581@gmail.com 